\title{
A Comprehensive Model of Glucose-Insulin Regulation Including Acute and Prolonged Effects of Physical Activity in Type 1 Diabetes
}

\author{
Julia Deichmann ${ }^{1,2}$, Sara Bachmann ${ }^{3,4}$, Marc Pfister ${ }^{5}$, Gabor \\ Szinnai $^{3,4}$, and Hans-Michael Kaltenbach ${ }^{1, *}$
}

${ }^{1}$ Department of Biosystems Science and Engineering and SIB Swiss Institute of Bioinformatics, ETH Zurich, Basel, Switzerland ${ }^{2}$ Life Science Zurich Graduate School, Zurich, Switzerland

${ }^{3}$ Department of Pediatric Endocrinology and Diabetology, University Children's Hospital Basel, Switzerland

${ }^{4}$ Department of Clinical Research, University Hospital Basel,

University of Basel, Basel, Switzerland

${ }^{5}$ Department of Pediatric Pharmacology and Pharmacometrics, University Children's Hospital Basel, University of Basel, Basel, Switzerland

*e-mail: michael.kaltenbach@bsse.ethz.ch

June 9, 2021

\begin{abstract}
Objective: For type 1 diabetic patients, accurate adjustment of insulin treatment to physical activity (PA) is a challenging open problem. Glucose uptake by the exercising muscles increases acutely, causing increased hepatic glucose production to maintain glucose homeostasis. Meanwhile, insulin sensitivity is elevated for a prolonged period to drive glycogen repletion during recovery. These processes strongly depend on PA duration and intensity, making their combined effects difficult to predict accurately. In this work, we develop a model of glucose-insulin regulation that captures PA from low to high intensity including acute and prolonged effects on glucose metabolism.

Methods: We extended an existing minimal model of glucose-insulin regulation to capture PA-driven changes in glucose metabolism. We incorporated the insulin-independent increase in glucose uptake and production, including the effects of glycogen depletion and of high-intensity PA on production. The model also captures the prolonged increase in insulin sensitivity.

Results: The model accurately predicts glucose dynamics of published data during short and prolonged PA of moderate to high intensity and during subsequent recovery. In-silico full-day studies elucidate the effects of timing, duration and intensity of PA and of insulin bolus reduction on
\end{abstract}


glucose levels during and after the activity.

Conclusion: The proposed model captures the blood glucose dynamics during all main PA regimes.

Significance: Mathematical models of glucose-insulin regulation are critical components of closed-loop insulin delivery and clinical decision support systems for achieving good glycemic control. The presented model shows potential for the development and assessment of algorithms targeting treatment adjustment to PA.

\section{Introduction}

Type 1 diabetes (T1D) results from autoimmune destruction of pancreatic $\beta$ cells. Affected patients depend on lifelong treatment by exogenous insulin to achieve good glycemic control.

Physical activity (PA) is beneficial for T1D patients and recommended in clinical guidelines [5,14], but fear of acute and late-onset (often nocturnal) hypoglycemia restrains many patients from exercising [7]. Preventing hypoglycemia by adjusting insulin treatment and nutrition is a major challenge due to the complexity of PA-driven changes in glucose metabolism, and current guidelines consider only coarse categories of glycemia, PA duration and intensity to determine the recommended adjustments $[1,13,37]$.

Exercise-induced processes occur on different time-scales and strongly depend on duration and intensity of PA [39]. During PA, the glucose demand by active muscles increases acutely and glucose uptake (GU) from plasma is upregulated. Simultaneously, hepatic glucose production (GP) by gluconeogenesis and glycogenolysis increases to maintain plasma glucose levels $[9,26]$. During prolonged PA, liver glycogen stores may deplete and GP cannot be maintained, causing a drop in glucose levels [19]. In contrast, GP may (initially) exceed GU and result in rising plasma glucose during high-intensity PA due to an increase in catecholamines and cortisol [32]. During recovery, insulin-independent GU and GP rates quickly return to their baseline levels [9]. However, insulin sensitivity can stay elevated for up to 48 hours after PA to replete liver glycogen stores and may cause late-onset hypoglycemia, including nocturnal hypoglycemia [33].

Mathematical models allow us to determine clinical parameters such as insulin sensitivity from an intravenous glucose tolerance test [6] and to better understand the glucose-insulin system in physiological detail [41]. They also play a critical role in the development of decision support systems and of closed-loop insulin delivery systems (artificial pancreas) [11,28, 29].

The need to include the impact of PA on glucose metabolism in diabetesrelated models has long been recognized $[13,38,42]$, but the prolonged and nonlinear effects during exercise and recovery pose formidable challenges. Existing models are usually restricted to moderate intensity and often focus exclusively on either short-term or prolonged effects of PA. To-date, no model simultaneously incorporates all acute and prolonged changes in glucose metabolism caused by exercise from low to high intensity, which limits the applicability to predict exercise-induced hypoglycemia and to determine accurate treatment adjustments.

A first extension of the Bergman minimal model [6] includes constant intensity exercise with acute increases in glucose uptake and production and eventual liver glycogen depletion, but not the elevated insulin sensitivity during 
recovery [40]. An alternative extension by Breton allows different exercise intensities for the insulin-independent increase in glucose clearance but not for insulin sensitivity, and does not consider glycogen depletion [8]. Starting from their earlier glucose-insulin model with carbohydrate intake, Dalla Man and coworkers integrate Breton's exercise model and add intensity-dependent insulin sensitivity $[15,16]$.

Two physiologically detailed models with exercise include glucose uptake and production, but no changes in insulin sensitivity [30], respectively glycogen depletion with increased hepatic glucose uptake after a meal to drive glycogen repletion after PA [21]. Based on the latter, a virtual patient population generator capturing PA has been proposed [36]. Furthermore, multi-scale models of tissue and organ systems connected by the circulatory system have been considered $[27,35]$.

Here, we propose a semi-mechanistic model of glucose-insulin regulation that captures the acute and prolonged changes in glucose metabolism during PA and subsequent recovery for low- to high-intensity exercise. We use a well-established two-compartment minimal model [12] as our core model for glucose-insulin dynamics and combine it with simple models of insulin kinetics [34] and carbohydrate absorption. We then incorporate the separate acute increase in glucose uptake and production - including depletion - and the prolonged rise in insulin sensitivity caused by PA. We use sigmoidal transfer functions to compactly describe some of the physiological changes. Our model accurately predicts blood glucose dynamics during and after PA and could be used to evaluate the associated hypoglycemia risk several hours post-exercise. Its modularity allows easy extensions of the insulin and meal submodels, which makes the developed model suitable for incorporation into both decision support systems ('exercise calculator' [13]) with bolus injections and pump-based artificial pancreas systems.

\section{Methods}

Our model consists of a core model including basic glucose-insulin regulation, insulin bolus kinetics, and meal absorption, and an exercise model capturing the PA-related changes in glucose metabolism.

\subsection{Core Model}

\subsubsection{Two-compartment minimal model}

The two-compartment minimal model [12] describes the impact of insulin and glucose on plasma glucose levels with compartment $Q_{1}[\mathrm{mg} / \mathrm{kg}]$ to represent glucose mass in plasma, and a remote compartment $Q_{2}[\mathrm{mg} / \mathrm{kg}]$ (Fig. 1). Plasma insulin promotes the disappearance of plasma glucose into liver and tissue, and suppresses hepatic glucose production via the dynamic state $X[1 / \mathrm{min}]$ :

$$
\begin{aligned}
\dot{X}(t)= & -p_{2} \cdot X(t)+p_{3} \cdot I(t) \\
\dot{Q}_{1}(t)= & -\left[p_{1}+X(t)\right] \cdot Q_{1}(t)-p_{4} \cdot Q_{1}(t) \\
& +p_{5} \cdot Q_{2}(t)+\left(p_{1}+X_{b}\right) \cdot Q_{1 b}+R a(t) \\
\dot{Q}_{2}(t)= & p_{4} \cdot Q_{1}(t)-p_{5} \cdot Q_{2}(t) \\
G(t)= & Q_{1}(t) / V_{g} .
\end{aligned}
$$




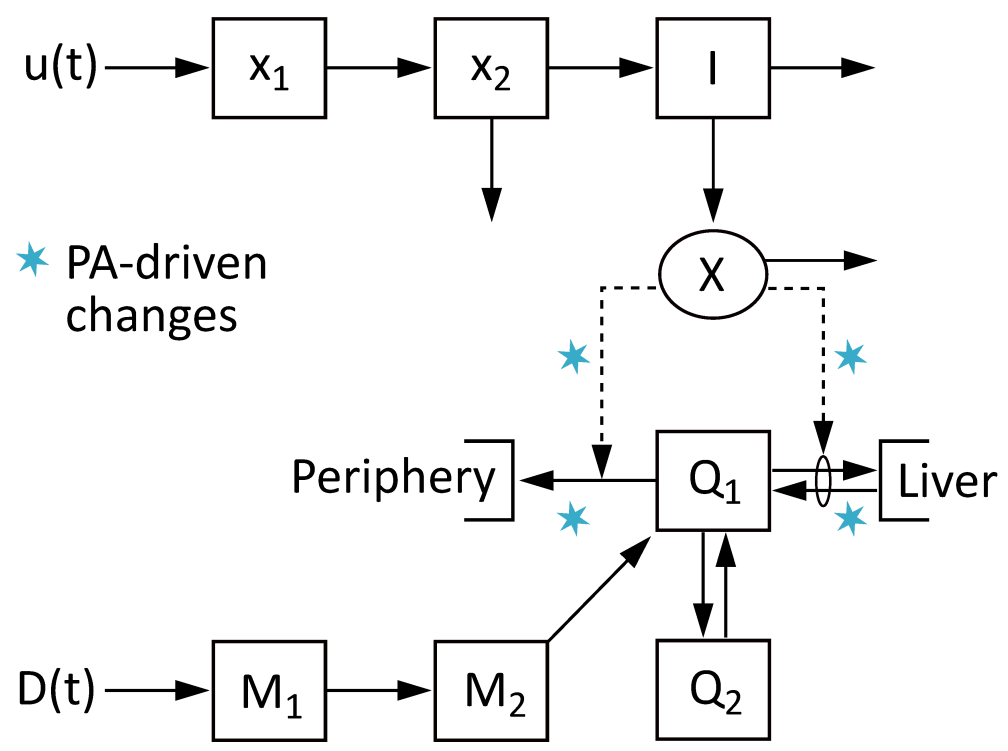

Figure 1: Schematic of the glucose-insulin model used to describe glucose $\left(Q_{1}\right)$ and insulin $(I)$ dynamics. It is based on the two-compartment minimal model [12], extended to capture plasma insulin kinetics [34] after injection $u$ and to capture the glucose response to a meal $D . X$ is a dynamic state related to insulin concentration. Processes affected by PA are marked with a blue asterisk.

Here, $I[\mu \mathrm{U} / \mathrm{ml}]$ is the plasma insulin concentration, $Q_{1 b}[\mathrm{mg} / \mathrm{kg}]$ denotes the basal level of glucose and $X_{b}$ the basal value of $X$ with $X_{b}=p_{3} / p_{2} \cdot I_{b}$, $R a[\mathrm{mg} / \mathrm{kg} / \mathrm{min}]$ is the rate of glucose appearance in plasma after a meal. $G$ $[\mathrm{mg} / \mathrm{dl}]$ is the plasma glucose concentration, $V_{g}[\mathrm{dl} / \mathrm{kg}]$ the glucose distribution volume and $p_{i}$ are rate parameters.

\subsubsection{Insulin kinetics model}

We use a model with two subcutaneous compartments of insulin masses $x_{1}$ and $x_{2}[\mu \mathrm{U}]$ and a plasma insulin compartment $I_{c}[\mu \mathrm{U} / \mathrm{ml}]$ (Fig. 1) to capture the resulting plasma insulin kinetics after a subcutaneous bolus injection [34]:

$$
\begin{aligned}
\dot{x}_{1}(t) & =-k_{1} \cdot x_{1}(t)+u(t) \\
\dot{x}_{2}(t) & =k_{1} \cdot x_{1}(t)-\left(k_{2}+k_{3}\right) \cdot x_{2}(t) \\
\dot{I}_{c}(t) & =\frac{k_{2}}{V_{i} \cdot B W} \cdot x_{2}(t)-k_{4} \cdot I_{c}(t) \\
I(t) & =I_{c}(t)+I_{b} .
\end{aligned}
$$

Insulin is injected into $x_{1}$ at rate $u[\mu \mathrm{U} / \mathrm{min}]$ and yields a rise $I_{c}$ in plasma insulin concentration $I$, adjusted for distribution volume $V_{i}[\mathrm{ml} / \mathrm{kg}]$ and bodyweight $B W[\mathrm{~kg}] . k_{i}$ are rate parameters and we assume a constant basal insulin level $I_{b}[\mu \mathrm{U} / \mathrm{ml}]$. 


\subsubsection{Meal absorption model}

We describe glucose appearance after a meal with a two-compartment absorption model:

$$
\begin{aligned}
\dot{M}_{1}(t) & =-m_{1} \cdot M_{1}(t)+D(t) \\
\dot{M}_{2}(t) & =m_{1} \cdot M_{1}(t)-m_{2} \cdot M_{2}(t) \\
R a(t) & =\frac{f \cdot m_{2}}{B W} \cdot M_{2}(t),
\end{aligned}
$$

where the ingested glucose, $D[\mathrm{mg} / \mathrm{min}]$, first passes through compartments $M_{1}[\mathrm{mg}]$ and $M_{2}[\mathrm{mg}]$ before being absorbed (Fig. 1). $R a[\mathrm{mg} / \mathrm{kg} / \mathrm{min}]$ defines the rate of glucose appearance in plasma as the fraction $f$ of absorbed glucose; $m_{i}$ are rate parameters.

\subsection{Exercise Model}

To incorporate exercise-driven changes, we replace the plasma glucose mass equation (1) with

$$
\begin{aligned}
\dot{Q}_{1}(t)= & -\left[p_{1}+r_{G U}(t)-\left[r_{G P}(t)-r_{\text {depl }}(t)\right]\right. \\
& +[1+Z(t)] \cdot X(t)] \cdot Q_{1}(t)-p_{4} \cdot Q_{1}(t) \\
& +p_{5} \cdot Q_{2}(t)+\left(p_{1}+X_{b}\right) \cdot Q_{1 b}+R a(t) .
\end{aligned}
$$

The rates $r_{G U}$ and $\left(r_{G P}-r_{d e p l}\right)$ provide the insulin-independent increase in GU and GP (including glycogen depletion), respectively, while $(1+Z)$ captures the PA-driven increase in insulin sensitivity.

We model smooth transitions between different exercise modes, e.g. lowand high-intensity PA, via the transfer function

$$
f(x ; p, n)=\frac{(x / p)^{n}}{1+(x / p)^{n}} .
$$

Although this transfer function does not offer a physiologically accurate description of the underlying molecular mechanisms, it enables us to keep the model simple, while incorporating the effects of different exercise regimes on glucose levels. This strategy has been used before to activate the exercise-driven increase in insulin sensitivity [8].

\subsubsection{Measure of exercise intensity and duration}

We consider movement via accelerometer (AC) counts, which we link to PA intensity $Y$ via a delay $\tau_{A C}[\mathrm{~min}]$ to allow initial adaptation to PA:

$$
\dot{Y}(t)=-\frac{1}{\tau_{A C}} \cdot Y(t)+\frac{1}{\tau_{A C}} \cdot A C(t) .
$$

Furthermore, we track PA duration $t_{P A}[\mathrm{~min}]$, integrated $\mathrm{AC}$ count $P A_{\text {int }}$ [counts] and time spent at high intensity $t_{H I}[\mathrm{~min}]$ :

$$
\begin{aligned}
\dot{t}_{P A}(t)= & f\left(A C ; a_{A C}, n_{2}\right)-\left[1-f\left(A C ; a_{A C}, n_{2}\right)\right] \cdot t_{P A}(t) \\
\dot{P A_{i n t}}(t)= & f\left(A C ; a_{A C}, n_{2}\right) \cdot A C(t) \\
& -\left[1-f\left(A C ; a_{A C}, n_{2}\right)\right] \cdot P A_{\text {int }}(t) \\
\dot{t}_{H I}(t)= & f\left(A C ; a_{H I}, n_{2}\right)-\left[1-f\left(A C ; a_{H I}, n_{2}\right)\right] \cdot t_{H I}(t),
\end{aligned}
$$


where transfer functions $f\left(A C ; a_{A C}, n_{2}\right)$ and $f\left(A C ; a_{H I}, n_{2}\right)$ capture the transition from rest to exercise, respectively from low to high intensity.

\subsubsection{Insulin sensitivity}

We model the rise in insulin sensitivity as

$$
\dot{Z}(t)=b \cdot f\left(Y ; a_{Y}, n_{1}\right) \cdot Y(t)-\frac{1}{\tau_{Z}} \cdot\left[1-f\left(Y ; a_{Y}, n_{1}\right)\right] \cdot Z(t),
$$

where $f\left(Y ; a_{Y}, n_{1}\right)$ defines the minimal intensity $Y$ considered as $\mathrm{PA} ; b$ and $\tau_{Z}$ are parameters. Insulin sensitivity increases proportionally with intensity during PA, stays elevated to drive glycogen repletion after PA, and then returns slowly to its baseline.

\subsubsection{Insulin-dependent glucose uptake and production}

The PA-driven, insulin-independent glucose uptake $\left(r_{G U}[1 / \mathrm{min}]\right)$ and production $\left(r_{G P}[1 / \mathrm{min}]\right)$ rates are given by

$$
\begin{aligned}
& \dot{r}_{G U}(t)=q_{1} \cdot f\left(Y ; a_{Y}, n_{1}\right) \cdot Y(t)-q_{2} \cdot r_{G U}(t) \\
& \dot{r}_{G P}(t)=q_{3} \cdot f\left(Y ; a_{Y}, n_{1}\right) \cdot Y(t)-q_{4} \cdot r_{G P}(t) .
\end{aligned}
$$

Both rates depend on the PA intensity and are used to model a quick-on, quick-off behavior with rate parameters $q_{i}$. Parameters $q_{3}$ and $q_{4}$ are modulated between low- (subscript 'LI') and high-intensity (subscript 'HI') values by a transfer function to smoothly transition between the two exercise regimes:

$$
\begin{aligned}
& q_{3}=\left[1-f\left(t_{H I} ; t_{p}, n_{2}\right)\right] \cdot q_{3 L I}+f\left(t_{H I} ; t_{p}, n_{2}\right) \cdot q_{3 H I} \\
& q_{4}=\left[1-f\left(t_{H I} ; t_{p}, n_{2}\right)\right] \cdot q_{4 L I}+f\left(t_{H I} ; t_{p}, n_{2}\right) \cdot q_{4 H I}
\end{aligned}
$$
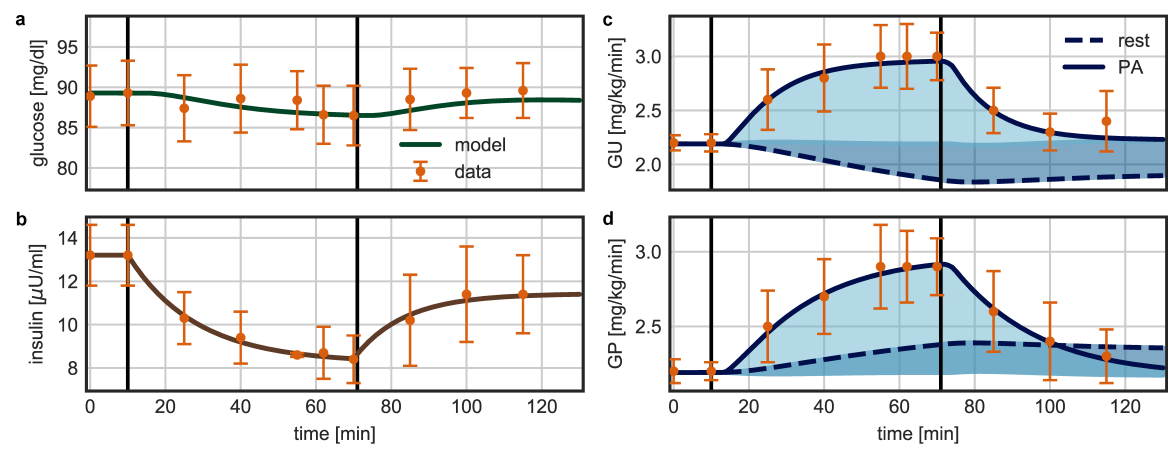

Figure 2: Data [44] and model prediction for $60 \mathrm{~min}$ of moderate-intensity $\mathrm{PA}$ (vertical lines) at $40 \% \mathrm{VO}_{2}{ }^{\mathrm{max}}$. (a) Model prediction of plasma glucose concentration. (b) Plasma insulin concentration. Model fit of (c) glucose uptake $(\mathrm{GU})$ and $(\mathrm{d})$ glucose production $(\mathrm{GP})$ rates during PA. The difference to resting rates is separated into contributions of insulin-dependent (dark shaded area) and insulin-independent (bright shaded area) changes in glucose metabolism due to PA. 
Table 1: Model Parameters

\begin{tabular}{|c|c|c|c|}
\hline Parameter & Value & Unit & From \\
\hline \multicolumn{4}{|c|}{ Glucose-insulin model } \\
\hline$p_{1}$ & 0.008 & $1 / \min$ & adapted [12] \\
\hline$p_{2}$ & 0.015 & $1 / \min$ & set manually \\
\hline$p_{3}$ & $2.47 \cdot 10^{-5}$ & $\mathrm{ml} / \mu \mathrm{U} 1 / \mathrm{min}^{2}$ & original [12] \\
\hline$p_{4}$ & 0.058 & $1 / \min$ & original $[12]$ \\
\hline$p_{5}$ & 0.0885 & $1 / \min$ & original $[12]$ \\
\hline$V_{g}$ & 1.289 & $\mathrm{dl} / \mathrm{kg}$ & original [12] \\
\hline \multicolumn{4}{|c|}{ Insulin model } \\
\hline$k_{1}$ & 0.022 & $1 / \min$ & set manually \\
\hline$k_{2}$ & 0.03 & $1 / \min$ & set manually \\
\hline$k_{3}$ & 0.0021 & $1 / \min$ & original [34] \\
\hline$k_{4}$ & 0.2 & $1 / \min$ & set manually \\
\hline$V_{i}$ & 125 & $\mathrm{ml} / \mathrm{kg}$ & original [34] \\
\hline \multicolumn{4}{|c|}{ Meal model } \\
\hline$m_{1}$ & 0.0115 & $1 / \min$ & estimated [23] \\
\hline$m_{2}$ & 0.0513 & $1 / \min$ & estimated [23] \\
\hline$f$ & 0.93 & dimensionless & estimated [23] \\
\hline \multicolumn{4}{|c|}{ Insulin sensitivity } \\
\hline$\tau_{A C}$ & 5 & $\min$ & physiology \\
\hline$\tau_{Z}$ & 600 & $\min$ & physiology \\
\hline$b$ & $1.68 \cdot 10^{-6}$ & $1 /$ count & estimated [43] \\
\hline \multicolumn{4}{|c|}{ Glucose uptake \& production } \\
\hline$\alpha$ & 0.36 & dimensionless & estimated [44] \\
\hline$q_{1}$ & $1.92 \cdot 10^{-7}$ & $1 /($ count $\cdot \min )$ & estimated [44] \\
\hline$q_{2}$ & 0.078 & $1 / \min$ & estimated [44] \\
\hline$q_{3 L I}$ & $1.19 \cdot 10^{-7}$ & $1 /($ count $\cdot \min )$ & estimated [44] \\
\hline$q_{4 L I}$ & 0.048 & $1 / \min$ & estimated [44] \\
\hline$q_{3 H I}$ & $2.51 \cdot 10^{-7}$ & $1 /($ count $\cdot \min )$ & estimated [25] \\
\hline$q_{4 H I}$ & 0.031 & $1 / \min$ & estimated [25] \\
\hline$q_{5}$ & 0.03 & $1 / \min$ & set manually \\
\hline \multicolumn{4}{|c|}{ Glycogen depletion } \\
\hline$\beta$ & 0.72 & dimensionless & estimated $[2]$ \\
\hline$q_{6}$ & 0.05 & $1 / \min$ & estimated [2] \\
\hline$a_{d e p l}$ & 0.0108 & $\min ^{2} /$ count & estimated [19] \\
\hline$b_{d e p l}$ & 180.6 & $\min$ & estimated [19] \\
\hline \multicolumn{4}{|c|}{ Transfer functions } \\
\hline$a_{Y}$ & 1500 & counts/min & \\
\hline$a_{A C}$ & 1000 & counts/min & \\
\hline$a_{H I}$ & 5600 & counts $/ \min$ & \\
\hline$t_{p}$ & 2 & $\min$ & \\
\hline$n_{1}$ & 20 & dimensionless & \\
\hline$n_{2}$ & 100 & dimensionless & \\
\hline
\end{tabular}




\subsubsection{Glycogen depletion}

We determine the time $t_{\text {depl }}[\mathrm{min}]$ until depletion of liver glycogen stores from the integrated $\mathrm{AC}$ count and PA duration:

$$
t_{d e p l}(t)=-a_{d e p l} \cdot \frac{P A_{i n t}(t)}{t_{P A}(t)}+b_{d e p l} .
$$

After depletion, GP decreases with rate $r_{\text {depl }}[1 / \mathrm{min}]$ :

$$
\begin{aligned}
\dot{r}_{d e p l}(t) & =q_{6} \cdot\left(f\left(t_{P A} ; t_{d e p l}, n_{1}\right) \cdot G P_{d m}(t)-r_{d e p l}(t)\right) \\
G P_{d m}(t) & =\beta \cdot\left(\frac{q_{3}}{q_{4}} \cdot Y(t)+G P_{b}\right)
\end{aligned}
$$

and the transfer function indicates whether exercise time exceeded $t_{d e p l}$. The maximum drop $G P_{d m}$ in GP is the sum of the PA-driven GP at steady state, $q_{3} / q_{4} \cdot Y(t)$, and the basal GP rate, $G P_{b} . q_{6}$ is a rate parameter and $\beta$ is the proportion of net hepatic glucose production attributed to glycogenolysis.

\subsection{Parameter Determination}

We obtained parameter values from literature or physiological knowledge when feasible. We estimated the remaining parameters from published data using least squares regression. Parameter values are given in Table 1.

We are aware that already the simple two-compartment minimal model of glucose-insulin regulation is known to be unidentifiable [12], and our model is therefore unlikely to be identifiable from blood glucose measurements alone. Nevertheless, we found that the reported parameter values enable accurate prediction of plasma glucose levels for a wide variety of experimental scenarios (see below). To calibrate our model, we have to rely on literature values and domain knowledge for some parameters. However, we calibrated the model parts related to physical activity based on published data sets. We separated parameters into process-specific sets and individually estimated these on data sets acquired during the corresponding exercise modes.

We used experimental data from different published studies to test if our calibrated model is able to accurately describe independent data. Importantly, these studies not only provide independent data not used in calibration of our model, but also investigated exercise at different intensities and for varying durations compared to the data sets used for parameter estimation. This enables us to investigate the general applicability of our model to various PA types and provides further validation of the intensity dependence given in the model.

We also tested whether model predictions qualitatively agree with clinical knowledge over an extended period of time by performing several full-day simulations with different PA scenarios and with and without insulin bolus adjustments.

\subsubsection{Core model parameters}

We slightly modified the original core model and manually adjusted insulin parameters to mimic the appearance of the plasma insulin peak after about $60 \mathrm{~min}$, typical for current fast-acting insulins. We estimated $m_{1}, m_{2}$ and $f$ of the meal model from plasma glucose measurements after a $75 \mathrm{~g}$ oral glucose load [23]. 


\subsubsection{Exercise model parameters}

We set the delay parameter $\tau_{A C}$ to $5 \mathrm{~min}[8]$ and chose a time constant $\tau_{Z}$ of 600 min such that insulin sensitivity stays elevated for up to $48 \mathrm{~h}$ in accordance with literature reports [33].

We estimated the increase in insulin sensitivity during PA (parameter $b$ ) from measurements of the insulin-dependent rate of glucose disappearance during rest and 100 min of cycling at $80 \% \mathrm{VO}_{2}{ }^{\max }$ in healthy subjects [43]. We converted $\% \mathrm{VO}_{2}{ }^{\max }$ to accelerometer count using

$$
\% V O_{2}^{\max }=0.0135 \cdot A C+1.7228
$$

estimated from simultaneous $\mathrm{AC}$ count and $\% \mathrm{VO}_{2}{ }^{\max }$ measurements for different types and intensities of PA [17].

We estimated the insulin-independent GU and GP parameters $q_{1}, q_{2}, q_{3 L I}$ and $q_{4 L I}$ from total GU and GP rates measured in healthy adults during 60 min of $\mathrm{PA}$ at $40 \% \mathrm{VO}_{2}{ }^{\max }$ [44]. We distinguished between resting and exercisedriven contributions by separating the net rate of glucose change at rest into endogenous glucose production and glucose uptake:

$$
\begin{aligned}
& G P(t)=\left(p_{1}+X_{b}\right) \cdot Q_{1 b}-\alpha \cdot\left[p_{1}+X(t)\right] \cdot Q_{1}(t) \\
& G U(t)=(1-\alpha) \cdot\left[p_{1}+X(t)\right] \cdot Q_{1}(t) .
\end{aligned}
$$

We estimated the high-intensity exercise parameters $q_{3 H I}$ and $q_{4 H I}$ from interstitial glucose measurements [25] of T1D patients performing 45 min of intervals at $82.5 \% \mathrm{VO}_{2}{ }^{\max }$ and introduced the parameter $q_{5}=0.03 \mathrm{~min}^{-1}$ to prevent a switch to low-intensity parameters during recovery.

We determined the time until hepatic glycogenolysis decreases due to glycogen depletion from reported depletion times for different intensities [19] (parameters $a_{\text {depl }}$ and $\left.b_{d e p l}\right)$. We estimated glycogen depletion parameters $\beta$ and $q_{6}$ from plasma glucose measurements [2] recorded during $3 \mathrm{~h}$ of cycling at $58 \%$ $\mathrm{VO}_{2}{ }^{\max }$ in healthy individuals, where we restricted $q_{6}$ to $0.05 \mathrm{~min}^{-1}$ to avoid an overshoot in GP after PA.

Finally, we enforce the transition from rest to PA between 1000 and 2000 counts $/ \mathrm{min}$ with parameters $a_{Y}=1500$ counts $/ \mathrm{min}$ and $n_{1}=20$ [17]. Accordingly, we defined $a_{A C}=1000$ counts $/ \mathrm{min}$ and $n_{2}=100$ to track duration and AC count immediately from the start of PA. High-intensity PA commences at $80 \% \mathrm{VO}_{2} \max (5800$ counts $/ \mathrm{min})$, and we set $a_{H I}=5600$ counts $/ \mathrm{min}$ and $t_{p}=$ 2 min for a transition between intensity regimes at $75 \%-80 \% \mathrm{VO}_{2}$ max.

\section{Results}

\subsection{Glucose Metabolism during Moderate-Intensity Exer- cise}

We first evaluated the effects of moderate-intensity PA on plasma glucose dynamics on a cycling session of $60 \mathrm{~min}$ at $40 \% \mathrm{VO}_{2}$ max , the data used for estimating the GU and GP rates [44]. Model and observed data are in very good agreement (Fig. 2).

Both GU and GP rates increase at the onset of PA, starting from a baseline level of $2.2 \mathrm{mg} / \mathrm{kg} / \mathrm{min}$. GU increases to $3.0 \mathrm{mg} / \mathrm{kg} / \mathrm{min}$ due to a higher uptake 
by the exercising muscles, while GP increases to $2.9 \mathrm{mg} / \mathrm{kg} / \mathrm{min}$ to meet the increased demand. During recovery, both rates return quickly to their preexercise levels.

In our model, we separate the PA-driven changes in GU and GP into insulindependent and -independent contributions. While insulin-independent GU and GP rise immediately at the beginning of PA and turn off quickly after the end of exercise, insulin sensitivity increases gradually during the activity and stays elevated during recovery, leading to a continued rise in GU and drop in GP compared to resting rates. Plasma insulin concentration decreases during PA in the healthy individual to counteract the effects of increased insulin sensitivity (while T1D patients must actively reduce the insulin dose), and increases again after PA to drive glycogen repletion. Glucose homeostasis is maintained throughout the activity, where plasma glucose levels only decrease slightly from $89.3 \mathrm{mg} / \mathrm{dl}$ to $86.5 \mathrm{mg} / \mathrm{dl}$, and return to previous levels after the activity.

We validated the calibrated exercise model on data from two additional independent studies in healthy subjects. In the first study [22], participants cycled for $60 \mathrm{~min}$ at $60 \% \mathrm{VO}_{2}$ max. Glucose levels decreased from $93.7 \mathrm{mg} / \mathrm{dl}$ to $86.5 \mathrm{mg} / \mathrm{dl}$ and remained constant during the first hour of recovery. In the second study [4], participants performed arm exercise on a cycle ergometer for 120 min at $30 \% \mathrm{VO}_{2}{ }^{\max }$ and glucose concentration remained stable throughout PA. We again observe good agreement between data and model predictions (Fig. $3)$.

\subsection{Glycogen Depletion during Prolonged Exercise}

To evaluate the dynamics of glycogen depletion, we compared model predictions to the data for $180 \mathrm{~min}$ of $\mathrm{PA}$ at $58 \% \mathrm{VO}_{2}{ }^{\max }$ previously used for parameter estimation [2]. During the first two hours of PA, plasma glucose concentration decreases slowly from $79.1 \mathrm{mg} / \mathrm{dl}$ to $64 \mathrm{mg} / \mathrm{dl}$. In the last $60 \mathrm{~min}$, GP drops below its pre-exercise levels due to depletion, causing glucose levels to drop quickly reaching $50.1 \mathrm{mg} / \mathrm{dl}$ at the end of PA. During recovery, GP returns to the baseline level and plasma glucose starts rising. The model correctly captures glycogen depletion after $136 \mathrm{~min}$ (Eq. 11) and the observed plasma glucose levels (Fig. 4a).

Importantly, we find good agreement of our model predictions with the observed data for the time before onset of depletion, even though we did not use this part of the data for model calibration. We also determined the time of depletion independently and again observe good agreement for these data.

For independent validation of the depletion model, we used a data set of plasma glucose levels measured in healthy adults during $240 \mathrm{~min}$ of cycling at $30 \% \mathrm{VO}_{2}{ }^{\max }$ [3]. At this intensity, depletion is reached after $158 \mathrm{~min}$. The model correctly predicts the drop in glucose levels from $81.3 \mathrm{mg} / \mathrm{dl}$ at the beginning to $56.2 \mathrm{mg} / \mathrm{dl}$ at the end of PA (Fig. 5).

\subsection{Glucose Dynamics during High-Intensity Exercise}

We used the previous data of high-intensity intervals at $82.5 \% \mathrm{VO}_{2}{ }^{\max }$ for 45 min to evaluate this part of the exercise model [25]. The initial glucose level is $180 \mathrm{mg} / \mathrm{dl}$ and increases during PA to a maximum of $201 \mathrm{mg} / \mathrm{dl}$ shortly after PA before returning to pre-exercise levels. GP increases from $3.7 \mathrm{mg} / \mathrm{kg} / \mathrm{min}$ 
bioRxiv preprint doi: https://doi.org/10.1101/2021.06.09.447693; this version posted June 10, 2021. The copyright holder for this preprint (which was not certified by peer review) is the author/funder, who has granted bioRxiv a license to display the preprint in perpetuity. It is made available under aCC-BY 4.0 International license.

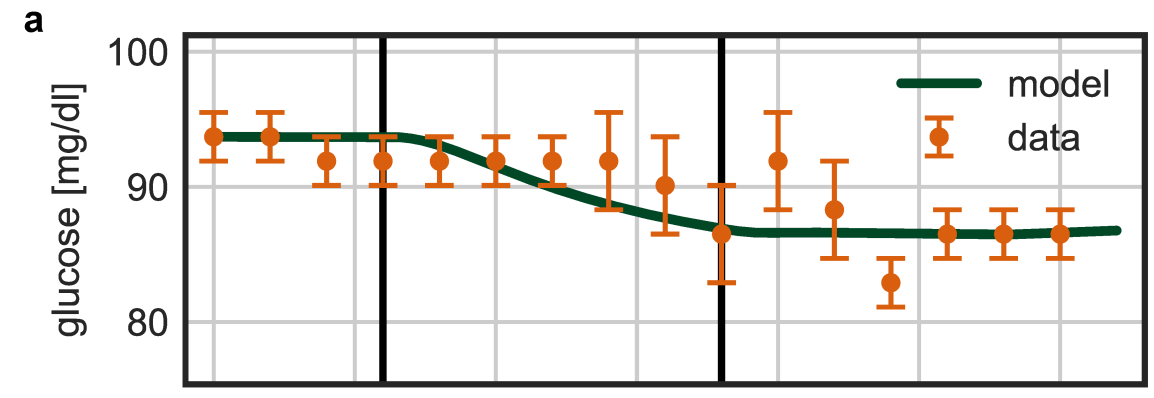

b

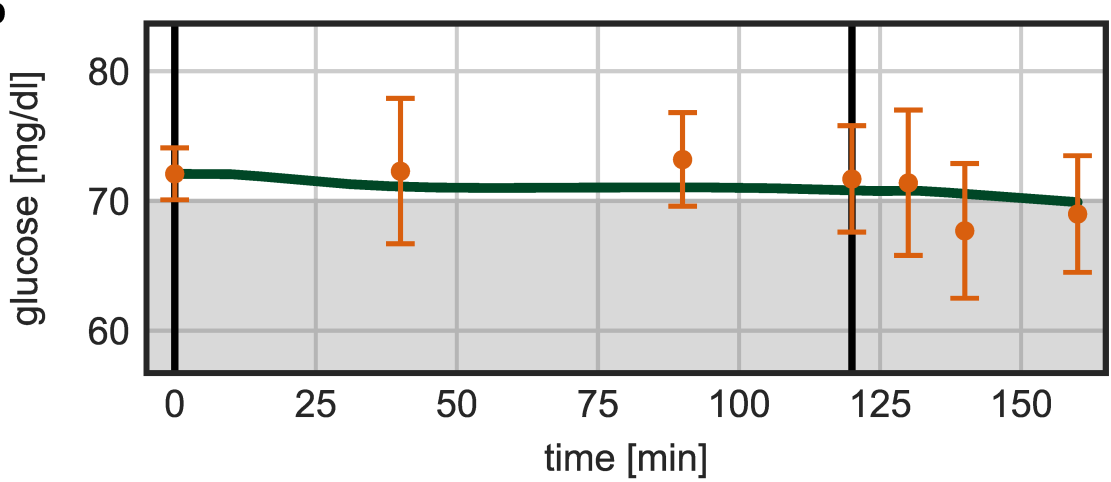

Figure 3: Validation of exercise model. (a) Model prediction and data [22] for 60 min of PA (vertical lines) at $60 \% \mathrm{VO}_{2}{ }^{\max }$. (b) Model prediction and data [4] for 120 min of $\mathrm{PA}$ at $30 \% \mathrm{VO}_{2}{ }^{\max }$.

at rest to $8.0 \mathrm{mg} / \mathrm{kg} / \mathrm{min}$ during $\mathrm{PA}$, while GU reaches $6.8 \mathrm{mg} / \mathrm{kg} / \mathrm{min}$. This dynamics is correctly reflected by the model (Fig. 4b).

\subsection{Full-Day Simulation of Glucose Dynamics in T1D}

Overall, our model adequately reflects experimental data for a range of exercise scenarios and generalizes to independent scenarios. We next considered its potential for long-term predictions for a full day of a T1D patient, consisting of three meals and corresponding insulin boluses. We defined twelve PA scenarios for this typical day: exercise in the morning or afternoon, of duration 60 or 180 min and with moderate $(30 \%$ or $60 \%)$ or high $\left(90 \% \mathrm{VO}_{2}{ }^{\max }\right)$ intensity (Fig. 6).

For moderate intensities, plasma glucose decreases during PA and slowly approaches resting glucose levels afterwards. For prolonged PA of $180 \mathrm{~min}$, depletion shows slightly earlier for an intensity of $60 \% \mathrm{VO}_{2}{ }^{\max }$ than for $30 \%$ $\mathrm{VO}_{2}{ }^{\max }$. Blood glucose decreases with increasing intensity and duration. Glucose levels are lower during the night for afternoon PA than for comparable morning PA, indicating higher risk for nocturnal hypoglycemia when exercise is performed later in the day.

Glucose concentration increases during high-intensity PA, but decreases to levels below those of moderate-intensity PA after the activity. The model thus confirms observations that high-intensity PA protects against hypoglycemia 
bioRxiv preprint doi: https://doi.org/10.1101/2021.06.09.447693; this version posted June 10, 2021. The copyright holder for this preprint (which was not certified by peer review) is the author/funder, who has granted bioRxiv a license to display the preprint in perpetuity. It is made available under aCC-BY 4.0 International license.
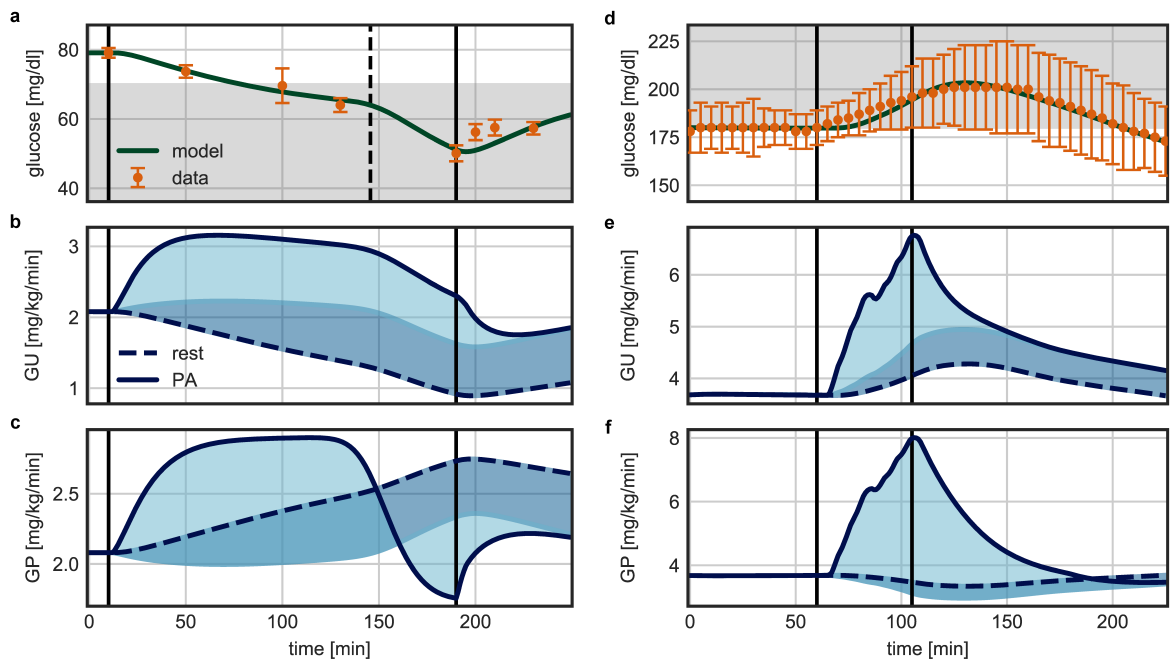

Figure 4: (a-c) Data [2] and model prediction for $180 \mathrm{~min}$ of PA (vertical lines) at $58 \% \mathrm{VO}_{2}{ }^{\max }$. (d-f) Data [25] and model prediction for $45 \mathrm{~min}$ of high-intensity $\mathrm{PA}$ at $82.5 \% \mathrm{VO}_{2}{ }^{\mathrm{max}}$. (a, d) Model fits of plasma glucose concentration, where the dashed vertical line indicates time of depletion. Model predictions of $(b, e)$ GU and (c, f) GP during PA. The difference to resting rates is separated into contributions of insulin-dependent (dark shaded area) and insulin-independent (bright shaded area) changes in glucose metabolism due to PA.

short-term [20], while the risk for late-onset hypoglycemia increases with higher intensity and duration of PA [24,31]. Furthermore, risk of nocturnal hypoglycemia is increased following afternoon compared to morning PA [18].

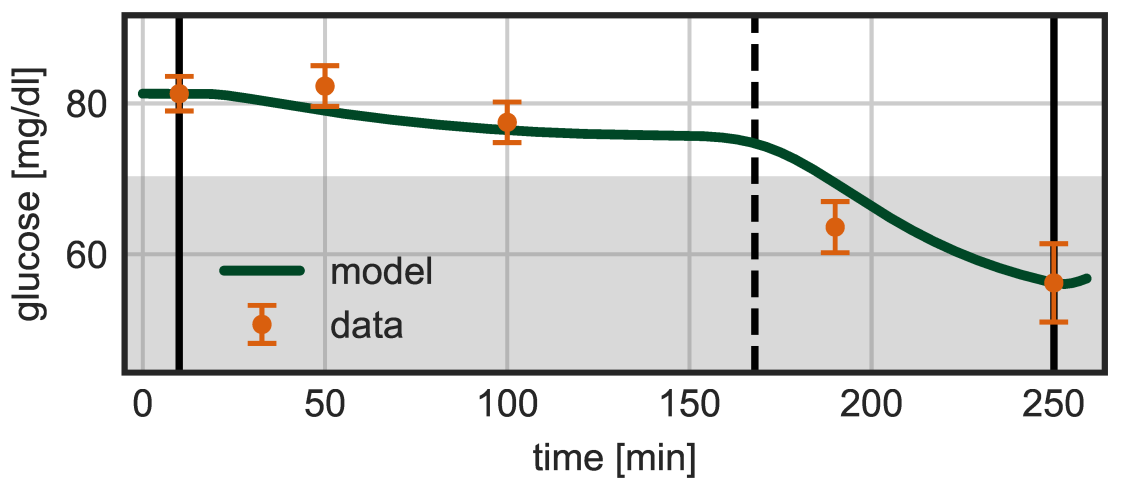

Figure 5: Model prediction and data [3] for $240 \mathrm{~min}$ of PA (vertical lines) at 30\% $\mathrm{VO}_{2}{ }^{\max }$ for validation of the exercise model. The dashed vertical line indicates time of depletion. 
bioRxiv preprint doi: https://doi.org/10.1101/2021.06.09.447693; this version posted June 10,2021 . The copyright holder for this preprint (which was not certified by peer review) is the author/funder, who has granted bioRxiv a license to display the preprint in perpetuity. It is made available under aCC-BY 4.0 International license.
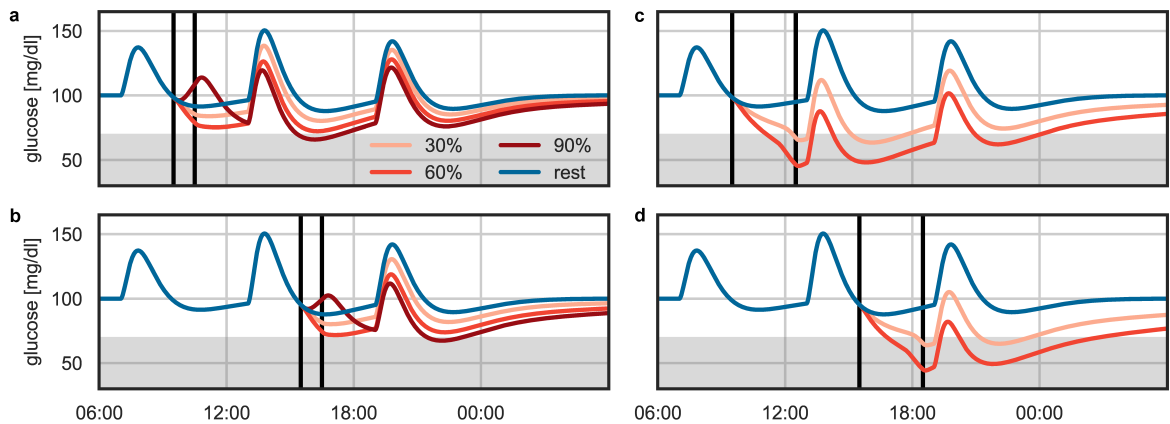

Figure 6: Full-day simulation of glucose dynamics at rest (blue), including three meals and corresponding insulin bolus injections. In addition, PA is performed (vertical lines) for (a, b) $60 \mathrm{~min}$ and (c, d) $180 \mathrm{~min}$ in the morning (a, c) and the afternoon $(b, d)$. Glucose dynamics are shown for moderate intensities of $30 \%$ and $60 \% \mathrm{VO}_{2}{ }^{\max }$ and for high-intensity $\mathrm{PA}$ at $90 \% \mathrm{VO}_{2}{ }^{\max }$.

\subsection{Insulin Reduction for Post-Exercise Meal Bolus}

Lastly, we studied reduction of the correction insulin bolus for a meal postexercise, a strategy successful in protecting against early-onset but not lateonset hypoglycemia [10]. We simulated PA in the morning from 10:00 to 12:00 and an insulin bolus for lunch at 13:00 either given in full, or reduced by $25 \%$ or $50 \%$ (Fig. 7 ).

As expected, plasma glucose is initially higher after lunch for reduced insulin compared to the full dose and is comparable to resting glucose levels. However, glucose concentrations are lower than resting levels throughout the night in all scenarios, independent of the bolus reduction. These results indicate that bolus reduction post-PA may protect against early-onset hypoglycemia, but does not target PA-induced late-onset hypoglycemia.

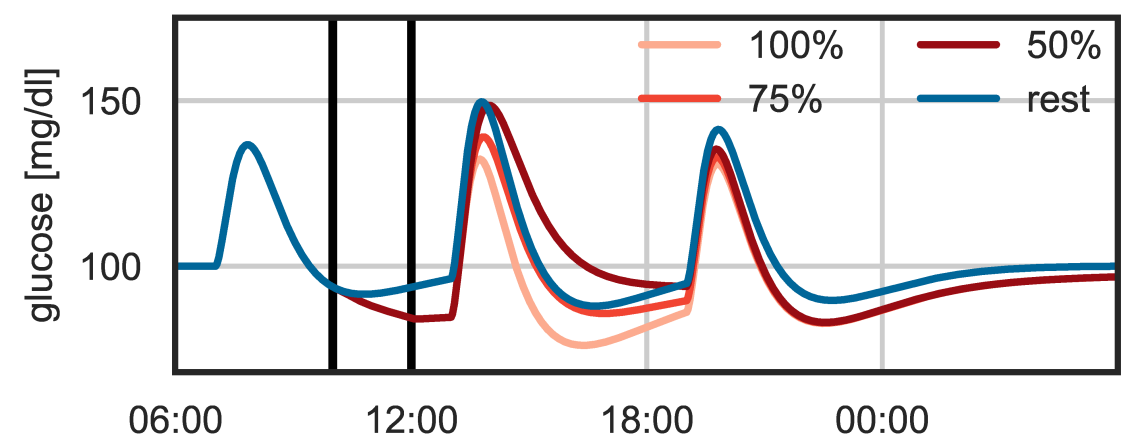

Figure 7: Full-day simulation with insulin reduction for post-PA meal bolus. Glucose dynamics are shown for rest (blue) and morning PA with a full (100\%), $75 \%$ and $50 \%$ insulin dose administered for lunch. 


\section{Discussion}

We presented a model of exercise-related changes in glucose-insulin regulation in type 1 diabetes. The model comprehensively covers acute and prolonged PA-driven processes from low to high intensity to capture changes in glucose dynamics during and after PA. We employ transfer functions to keep the model compact while allowing transitions between different exercise regimes.

Our model quantitatively describes exercise-driven changes in insulin sensitivity, GU and GP for all exercise regimes and allows for varying intensity throughout the activity. It describes the prolonged rise in insulin sensitivity that drives glycogen repletion during recovery, a main cause of late-onset hypoglycemia. We further modeled the insulin-independent acute rise in GU during the activity and the simultaneous increase in hepatic GP responsible for meeting the increased energy demand of the exercising muscles. To capture glucose metabolism during prolonged PA, we added the reduction in GP due to liver glycogen depletion. We also included GP increases during high-intensity PA.

Our model accurately reflects glucose dynamics for low- to high-intensity PA during the activity and the following recovery period. We validated model predictions on independent data of moderate-intensity PA, with and without depletion. No additional data were available for independent validation of the high-intensity model extension.

The developed model includes simple modules for insulin bolus injections and meal intake, which makes it suitable for full-day simulations with simple meals.

To evaluate the long-term prediction capabilities of the model, we presented full-day simulations with different PA scenarios. For moderate-intensity PA, model predictions agree with the observations that glucose levels decrease with intensity and duration. In addition, we correctly predict that blood glucose is lower during the night following afternoon compared to morning PA, which is associated with a higher risk for nocturnal hypoglycemia. Lastly, the model successfully captures the blood glucose rise during high-intensity PA, and the subsequent decrease below levels reached with comparable moderate-intensity PA several hours after the activity.

Our determination of the model parameters suffers from two drawbacks: first, we only identify some of the parameters directly from data, but have to rely on domain knowledge and literature values for other parameters. We partially addressed this problem by testing model predictions on independent data sets, but cannot ensure that the individual model parts are physiologically accurate. Second, the available data sets only provide average glucose responses over several study participants. For future application of the model, it would be beneficial to use individual patient data for subject-specific model adjustment and to assess inter-patient variability. Based on these results, an in-silico patient population could be generated and applied for realistic simulation studies, in which the whole range of potential glucose outcomes would be covered.

\section{Conclusion}

Summarizing, our comprehensive model of glucose-insulin regulation captures the acute and prolonged effects of low- to high-intensity PA on glucose metabolism. 
In addition, it includes meal intake and insulin injection kinetics, making it wellsuited to describe glucose dynamics during everyday life. Hence, the model can be used to predict blood glucose during PA and recovery and to evaluate the impact of PA on glucose levels acutely and up to several hours after the activity in practical scenarios. It can further be used to compare different exercise types and the associated hypoglycemia risk within one framework. We anticipate that our model finds applications as an 'exercise calculator' for clinical decision support [13] to assist patients and clinicians achieve good glycemic control after exercise, as well as for improving control algorithms for closed-loop insulin delivery. Its modular nature makes it easily adaptable to other forms of insulin delivery, in particular insulin pumps, and readily allows extension of our comparatively simple meal model to more complex meals.

We evaluated the model's performance on real data, but further validation of the model components on the individual patient level is warranted before application in a clinical setting.

\section{Acknowledgment}

We thank Jörg Stelling, Gilbert Koch and Tamara van Donge for helpful feedback and discussions.

\section{References}

[1] Peter Adolfsson, Michael C. Riddell, Craig E. Taplin, Elizabeth A. Davis, Paul A. Fournier, Francesca Annan, Andrea E. Scaramuzza, Dhruvi Hasnani, and Sabine E. Hofer. ISPAD Clinical Practice Consensus Guidelines 2018: Exercise in children and adolescents with diabetes. Pediatric Diabetes, 19(June):205-226, 2018.

[2] G. Ahlborg and P. Felig. Lactate and glucose exchange across the forearm, legs, and splanchnic bed during and after prolonged leg exercise. Journal of Clinical Investigation, 69(1):45-54, 1982.

[3] G. Ahlborg, P. Felig, L. Hagenfeldt, R. Hendler, and J. Wahren. Substrate turnover during prolonged exercise in man. Splanchnic and leg metabolism of glucose, free fatty acids, and amino acids. Journal of Clinical Investigation, 53(4):1080-1090, 1974.

[4] G. Ahlborg, J. Wahren, and P. Felig. Splanchnic and peripheral glucose and lactate metabolism during and after prolonged arm exercise. Journal of Clinical Investigation, 77(3):690-699, 1986.

[5] American Diabetes Association. 4. Foundations of care: Education, nutrition, physical activity, smoking cessation, psychosocial care, and immunization. Diabetes Care, 38(January):S20-S30, 2015.

[6] Richard N. Bergman, Y. Ziya Ider, Charles R. Bowden, and Claudio Cobelli. Quantitative estimation of insulin sensitivity. American Journal of Physiology, 236(6):E667-E677, 1979. 
[7] Anne Sophie Brazeau, Rémi Rabasa-Lhoret, Irene Strychar, and Hortensia Mircescu. Barriers to physical activity among patients with type 1 diabetes. Diabetes Care, 31(11):2108-2109, 2008.

[8] Marc D. Breton. Physical activity-the major unaccounted impediment to closed loop control. Journal of Diabetes Science and Technology, 2(1):169 $174,2008$.

[9] Raul C. Camacho, Pietro Galassetti, Stephen N. Davis, and David H. Wasserman. Glucoregulation during and after exercise in health and insulin-dependent diabetes. Exercise and sport sciences reviews, 33(1):1723, 2005.

[10] Matthew D. Campbell, Mark Walker, Michael I. Trenell, Djordje G. Jakovljevic, Emma J. Stevenson, Richard M. Bracken, Stephen C. Bain, and Daniel J. West. Large pre-and postexercise rapid-acting insulin reductions preserve glycemia and prevent early- but not late-onset hypoglycemia in patients with type 1 diabetes. Diabetes Care, 36(8):2217-2224, 2013.

[11] Ali Cinar and Kamuran Turksoy. Advances in Artificial Pancreas Systems - Adaptive and Multivariable Predictive Control. Springer, 2017.

[12] Claudio Cobelli, Andrea Caumo, and Matteo Omenetto. Minimal model SG overestimation and SI underestimation: improved accuracy by a Bayesian two-compartment model. The American journal of physiology, 277(3 Pt 1):E481-E488, 1999.

[13] Sheri R. Colberg, Remmert Laan, Eyal Dassau, and David Kerr. Physical activity and type 1 diabetes: Time for a rewire? Journal of Diabetes Science and Technology, 9(3):609-618, 2015.

[14] Sheri R. Colberg, Ronald J. Sigal, Jane E. Yardley, Michael C. Riddell, David W. Dunstan, Paddy C. Dempsey, Edward S. Horton, Kristin Castorino, and Deborah F. Tate. Physical activity/exercise and diabetes: A position statement of the American Diabetes Association. Diabetes Care, 39(11):2065-2079, 2016.

[15] Chiara Dalla Man, Marc D. Breton, and Claudio Cobelli. Physical Activity into the Meal Glucose-Insulin Model of Type 1 Diabetes: In Silico Studies. Journal of Diabetes Science and Technology, 3(1):56-67, 2009.

[16] Chiara Dalla Man, Robert A. Rizza, and Claudio Cobelli. Meal simulation model of the glucose- insulin system. IEEE Trans Biom Engin, 54(10):1740-1749, 2007.

[17] Kelly R. Evenson, Diane J. Catellier, Karminder Gill, Kristin S. Ondrak, and Robert G. McMurray. Calibration of two objective measures of physical activity for children. Journal of Sports Sciences, 26(14):1557-1565, 2008.

[18] Ana Maria Gomez, Claudia Gomez, Pablo Aschner, Angelica Veloza, Oscar Munoz, Claudia Rubio, and Santiago Vallejo. Effects of performing morning versus afternoon exercise on glycemic control and hypoglycemia frequency in type 1 diabetes patients on sensor-augmented insulin pump therapy. Journal of Diabetes Science and Technology, 9(3):619-624, 2015. 
[19] Javier T. Gonzalez, Cas J. Fuchs, James A. Betts, and Luc J. C. van Loon. Liver glycogen metabolism during and after prolonged endurance-type exercise. American Journal of Physiology - Endocrinology And Metabolism, 311(3):E543-E553, 2016.

[20] KJ Guelfi, TW Jones, and PA Fournier. Intermittent high-intensity exercise does not increase the risk of early postexercise hypoglycemia in individuals with type 1 diabetes. Diabetes Care, 28(2):416-418 3p, 2005.

[21] M. Hernández-Ordoñez and D. U. Campos-Delgado. An extension to the compartmental model of type 1 diabetic patients to reproduce exercise periods with glycogen depletion and replenishment. Journal of Biomechanics, 41(4):744-752, 2008.

[22] I. B. Hirsch, J. C. Marker, L. J. Smith, R. J. Spina, C. A. Parvin, J. O. Holloszy, and P. E. Cryer. Insulin and glucagon in prevention of hypoglycemia during exercise in humans. American Journal of Physiology Endocrinology and Metabolism, 260(5 23-5), 1991.

[23] R. A. Jackson, P. M. Blix, J. A. Matthews, L. M. Morgan, A. H. Rubenstein, and J. D.N. Nabarro. Comparison of peripheral glucose uptake after oral glucose loading and a mixed meal. Metabolism, 32(7):706-710, 1983.

[24] Jason R. Jaggers, Kristi M. King, Sara E. Watson, and Kupper A. Wintergerst. Predicting Nocturnal Hypoglycemia with Measures of Physical Activity Intensity in Adolescent Athletes with Type 1 Diabetes. Diabetes Technology and Therapeutics, 21(7):406-408, 2019.

[25] Dilshani C. Jayawardene, Sybil A. McAuley, Jodie C. Horsburgh, André La Gerche, Alicia J. Jenkins, Glenn M. Ward, Richard J. MacIsaac, Timothy J. Roberts, Benyamin Grosman, Natalie Kurtz, Anirban Roy, and David N. O'Neal. Closed-loop insulin delivery for adults with type 1 diabetes undertaking high-intensity interval exercise versus moderate-intensity exercise: A randomized, crossover study. Diabetes Technology and Therapeutics, 19(6):340-348, 2017.

[26] Thomas E. Jensen and Erik A. Richter. Regulation of glucose and glycogen metabolism during and after exercise. Journal of Physiology, 590(5):10691076, 2012.

[27] Jaeyeon Kim, Gerald M. Saidel, and Marco E. Cabrera. Multi-scale computational model of fuel homeostasis during exercise: Effect of hormonal control. Annals of Biomedical Engineering, 35(1):69-90, 2007.

[28] Boris Kovatchev. Automated closed-loop control of diabetes: the artificial pancreas. Bioelectronic Medicine, 4(1):1-12, 2018.

[29] Boris Kovatchev. A Century of Diabetes Technology: Signals, Models, and Artificial Pancreas Control. Trends in Endocrinology and Metabolism, 30(7):432-444, 2019.

[30] Philip J. Lenart and Robert S. Parker. Modeling exercise effects in type I diabetic patients, volume 35. IFAC, 2002. 
[31] Alberto Maran, Paola Pavan, Barbara Bonsembiante, Erica Brugin, Andrea Ermolao, Angelo Avogaro, and Marco Zaccaria. Continuous glucose monitoring reveals delayed nocturnal hypoglycemia after intermittent highintensity exercise in nontrained patients with type 1 diabetes. Diabetes Technology and Therapeutics, 12(10):763-768, 2010.

[32] Errol B. Marliss and Mladen Vranic. Intense exercise has unique effects on both insulin release and its roles in glucoregulation: Implications for diabetes. Diabetes, 51(SUPPL.):271-283, 2002.

[33] Joram D. Mul, Kristin I. Stanford, Michael F. Hirshman, and Laurie J. Goodyear. Exercise and Regulation of Carbohydrate Metabolism. Progress in Molecular Biology and Translational Science, 135:17-37, 2015.

[34] Gianluca Nucci and Claudio Cobelli. Models of subcutaneous insulin kinetics. A critical review. Computer Methods and Programs in Biomedicine, 62(3):249-257, 2000.

[35] Maria Concetta Palumbo, Micaela Morettini, Paolo Tieri, Fasma Diele, Massimo Sacchetti, and Filippo Castiglione. Personalizing physical exercise in a computational model of fuel homeostasis. PLoS Computational Biology, 14(4):1-23, 2018.

[36] Navid Resalat, Joseph El Youssef, Nichole Tyler, Jessica Castle, and Peter G. Jacobs. A statistical virtual patient population for the glucoregulatory system in type 1 diabetes with integrated exercise model. Plos One, 14(7):e0217301, 2019.

[37] Michael C. Riddell, Ian W. Gallen, Carmel E. Smart, Craig E. Taplin, Peter Adolfsson, Alistair N. Lumb, Aaron Kowalski, Remi Rabasa-Lhoret, Rory J. McCrimmon, Carin Hume, Francesca Annan, Paul A. Fournier, Claudia Graham, Bruce Bode, Pietro Galassetti, Timothy W. Jones, Iñigo San Millán, Tim Heise, Anne L. Peters, Andreas Petz, and Lori M. Laffel. Exercise management in type 1 diabetes: a consensus statement. The Lancet Diabetes and Endocrinology, 5(5):377-390, 2017.

[38] Michael C. Riddell, Dessi P. Zaharieva, Loren Yavelberg, Ali Cinar, and Veronica K. Jamnik. Exercise and the Development of the Artificial Pancreas: One of the More Difficult Series of Hurdles. Journal of diabetes science and technology, 9(6):1217-1226, 2015.

[39] J.A. Romijn, E.F. Coyle, L.S. Sidossis, A. Gastaldelli, J.F. Horowitz, E. Endert, and R.R. Wolfe. Regulation of endogenous fat and carbohydrate metabolism in relation to exercise intensity and duration. The American Journal of Physiology, 265(3p1):380-391, 1993.

[40] Anirban Roy and Robert S. Parker. Dynamic Modeling of Exercise Effects on Plasma Glucose and Insulin Levels. Journal of Diabetes Science and Technology, 1(3):338-347, 2007.

[41] John Thomas Sorensen. A physiologic model of glucose metabolism in man and its use to design and assess improved insulin therapies for diabetes. PhD thesis, Massachusetts Institute of Technology, 1978. 
[42] Sémah Tagougui, Nadine Taleb, Joséphine Molvau, Élisabeth Nguyen, Marie Raffray, and Rémi Rabasa-Lhoret. Artificial Pancreas Systems and Physical Activity in Patients with Type 1 Diabetes: Challenges, Adopted Approaches, and Future Perspectives. Journal of Diabetes Science and Technology, 13(6):1077-1090, 2019.

[43] D. H. Wasserman, R. J. Geer, D. E. Rice, D. Bracy, P. J. Flakoll, L. L. Brown, J. O. Hill, and N. N. Abumrad. Interaction of exercise and insulin action in humans. American Journal of Physiology-Endocrinology and Metabolism, 260(1):E37-E45, 1991.

[44] R. R. Wolfe, E. R. Nadel, J. H.F. Shaw, and L. A. Stephenson. Role of changes in insulin and glucagon in glucose homeostasis in exercise. Journal of Clinical Investigation, 77(3):900-907, 1986. 